PROCEEDINGS OF THE

AMERICAN MATHEMATICAL SOCIETY

Volume 131, Number 8, Pages 2501-2504

S 0002-9939(02)06886-7

Article electronically published on November 13, 2002

\title{
MULTI-QUADRATIC MAPPINGS IN BANACH SPACES
}

\author{
CHUN-GIL PARK
}

(Communicated by Joseph A. Ball)

\begin{abstract}
We prove the stability of multi-quadratic functional equations in Banach spaces.
\end{abstract}

\section{Stability of multi-quadratic Functional EQUATIOnS IN BANACH SPACES}

Let $E_{1}$ and $E_{2}$ be Banach spaces with norms $\|\cdot\|$ and $\|\cdot\|$, respectively. Consider $f: E_{1} \rightarrow E_{2}$ to be a mapping such that $f(t x)$ is continuous in $t \in \mathbb{R}$ for each fixed $x \in E_{1}$. Assume that there exist constants $\epsilon \geq 0$ and $p \in[0,1)$ such that

$$
\|f(x+y)-f(x)-f(y)\| \leq \epsilon\left(\|x\|^{p}+\|y\|^{p}\right)
$$

for all $x, y \in E_{1}$. Th.M. Rassias [2] showed that there exists a unique $\mathbb{R}$-linear mapping $T: E_{1} \rightarrow E_{2}$ such that

$$
\|f(x)-T(x)\| \leq \frac{2 \epsilon}{2-2^{p}}\|x\|^{p}
$$

for all $x \in E_{1}$.

In [1], the author showed the stability of multilinear functional equations in Banach modules over a unital $C^{*}$-algebra.

Throughout this paper, let $\mathcal{B}_{i}$ be normed spaces for $i=1, \cdots, d$. Let $\mathcal{D}$ be a Banach space with norm $\|\cdot\|$.

The main purpose of this paper is to prove the stability of multi-quadratic functional equations in Banach spaces.

Received by the editors March 22, 2002.

2000 Mathematics Subject Classification. Primary 47B48, 39B72.

Key words and phrases. Banach space, stability, multi-quadratic functional equation.

This work was supported by grant No. 1999-2-102-001-3 from the interdisciplinary research program year of the KOSEF. 
For a given mapping $f: \prod_{i=1}^{d} \mathcal{B}_{i} \rightarrow \mathcal{D}$, we set

$$
\begin{aligned}
& D f\left(x_{1}, y_{1}, \cdots, x_{d}, y_{d}\right):=\sum_{i=1}^{d} f\left(x_{1}, \cdots, x_{i-1}, x_{i}+y_{i}, x_{i+1}, \cdots, x_{d}\right) \\
&+\sum_{i=1}^{d} f\left(x_{1}, \cdots, x_{i-1}, x_{i}-y_{i}, x_{i+1}, \cdots, x_{d}\right) \\
&-2 d f\left(x_{1}, \cdots, x_{i}, \cdots, x_{d}\right)-\sum_{i=1}^{d} 2 f\left(x_{1}, \cdots, x_{i-1}, y_{i}, x_{i+1}, \cdots, x_{d}\right)
\end{aligned}
$$

for all $\left(x_{1}, \cdots, x_{d}\right),\left(y_{1}, \cdots, y_{d}\right) \in \prod_{i=1}^{d} \mathcal{B}_{i}$.

Theorem 1. Let $f: \prod_{i=1}^{d} \mathcal{B}_{i} \rightarrow \mathcal{D}$ be a mapping for which there exists a function $\varphi: \prod_{i=1}^{d} \mathcal{B}_{i}^{2} \rightarrow[0, \infty)$ such that

$$
\widetilde{\varphi}\left(x_{1}, y_{1}, \cdots, x_{d}, y_{d}\right):=\sum_{j=0}^{\infty} \sum_{i=1}^{d} \frac{1}{4^{i+j d}} \varphi\left(2^{j+1} x_{1}, 0, \cdots, 2^{j+1} x_{i-1}, 0,\right.
$$

$$
\begin{aligned}
& \left.2^{j} x_{i}, 2^{j} y_{i}, 2^{j} x_{i+1}, 0, \cdots, 2^{j} x_{d}, 0\right)<\infty, \\
\left\|D f\left(x_{1}, y_{1}, \cdots, x_{d}, y_{d}\right)\right\| & \leq \varphi\left(x_{1}, y_{1}, \cdots, x_{d}, y_{d}\right)
\end{aligned}
$$

for all $\left(x_{1}, \cdots, x_{d}\right),\left(y_{1}, \cdots, y_{d}\right) \in \prod_{i=1}^{d} \mathcal{B}_{i}$. Assume that $f\left(x_{1}, \cdots, x_{d}\right)=0$ if $x_{i}=0$ for any $i=1, \cdots, d$. Then there exists a unique multi-quadratic mapping $M: \prod_{i=1}^{d} \mathcal{B}_{i} \rightarrow \mathcal{D}$ such that

$$
\left\|f\left(x_{1}, \cdots, x_{d}\right)-M\left(x_{1}, \cdots, x_{d}\right)\right\| \leq \widetilde{\varphi}\left(x_{1}, x_{1}, \cdots, x_{d}, x_{d}\right)
$$

for all $\left(x_{1}, \cdots, x_{d}\right) \in \prod_{i=1}^{d} \mathcal{B}_{i}$.

Proof. For each fixed $i$, let $y_{1}=\cdots=y_{i-1}=y_{i+1}=\cdots=y_{d}=0$ and $y_{i}=x_{i}$ in (ii). Then we get

$$
\begin{aligned}
\| f\left(x_{1}, \cdots, x_{i-1}, 2 x_{i}, x_{i+1}, \cdots, x_{d}\right) & -4 f\left(x_{1}, \cdots, x_{i-1}, x_{i}, x_{i+1}, \cdots, x_{d}\right) \| \\
& \leq \varphi\left(x_{1}, 0, \cdots, x_{i-1}, 0, x_{i}, x_{i}, x_{i+1}, 0, \cdots, x_{d}, 0\right)
\end{aligned}
$$

for all $\left(x_{1}, \cdots, x_{d}\right) \in \prod_{i=1}^{d} \mathcal{B}_{i}$. So one can obtain

$$
\begin{array}{r}
\left\|f\left(x_{1}, \cdots, x_{i-1}, x_{i}, x_{i+1}, \cdots, x_{d}\right)-\frac{1}{4} f\left(x_{1}, \cdots, x_{i-1}, 2 x_{i}, x_{i+1}, \cdots, x_{d}\right)\right\| \\
\leq \frac{1}{4} \varphi\left(x_{1}, 0, \cdots, x_{i-1}, 0, x_{i}, x_{i}, x_{i+1}, 0, \cdots, x_{d}, 0\right)
\end{array}
$$

for all $\left(x_{1}, \cdots, x_{d}\right) \in \prod_{i=1}^{d} \mathcal{B}_{i}$. Hence

$$
\begin{aligned}
\| \frac{1}{4^{i-1}} f\left(2 x_{1}, \cdots, 2 x_{i-1},\right. & \left.x_{i}, \cdots, x_{d}\right)-\frac{1}{4^{i}} f\left(2 x_{1}, \cdots, 2 x_{i}, x_{i+1}, \cdots, x_{d}\right) \| \\
\leq & \frac{1}{4^{i}} \varphi\left(2 x_{1}, 0, \cdots, 2 x_{i-1}, 0, x_{i}, x_{i}, x_{i+1}, 0, \cdots, x_{d}, 0\right)
\end{aligned}
$$


for all $\left(x_{1}, \cdots, x_{d}\right) \in \prod_{i=1}^{d} \mathcal{B}_{i}$. Thus

$$
\begin{aligned}
\| f\left(x_{1}, \cdots, x_{d}\right)- & \frac{1}{4^{d}} f\left(2 x_{1}, \cdots, 2 x_{d}\right) \| \\
& \leq \sum_{i=1}^{d} \frac{1}{4^{i}} \varphi\left(2 x_{1}, 0, \cdots, 2 x_{i-1}, 0, x_{i}, x_{i}, x_{i+1}, 0, \cdots, x_{d}, 0\right)
\end{aligned}
$$

for all $\left(x_{1}, \cdots, x_{d}\right) \in \prod_{i=1}^{d} \mathcal{B}_{i}$. We also get

$$
\begin{aligned}
& \left\|\frac{1}{4^{j d}} f\left(2^{j} x_{1}, \cdots, 2^{j} x_{d}\right)-\frac{1}{4^{(j+1) d}} f\left(2^{j+1} x_{1}, \cdots, 2^{j+1} x_{d}\right)\right\| \\
& \quad \leq \sum_{i=1}^{d} \frac{1}{4^{i+j d}} \varphi\left(2^{j+1} x_{1}, 0, \cdots, 2^{j+1} x_{i-1}, 0,2^{j} x_{i}, 2^{j} x_{i}, 2^{j} x_{i+1}, 0, \cdots, 2^{j} x_{d}, 0\right)
\end{aligned}
$$

for all $\left(x_{1}, \cdots, x_{d}\right) \in \prod_{i=1}^{d} \mathcal{B}_{i}$. So

$$
\begin{aligned}
\| f\left(x_{1}, \cdots, x_{d}\right) & -\frac{1}{4^{n d}} f\left(2^{n} x_{1}, \cdots, 2^{n} x_{d}\right) \| \leq \sum_{j=0}^{n-1} \sum_{i=1}^{d} \frac{1}{4^{i+j d}} \\
& \times \varphi\left(2^{j+1} x_{1}, 0, \cdots, 2^{j+1} x_{i-1}, 0,2^{j} x_{i}, 2^{j} x_{i}, 2^{j} x_{i+1}, 0, \cdots, 2^{j} x_{d}, 0\right)
\end{aligned}
$$

for all $\left(x_{1}, \cdots, x_{d}\right) \in \prod_{i=1}^{d} \mathcal{B}_{i}$.

For each $i=1, \cdots, d$, let $x_{i}$ be an element in $\mathcal{B}_{i}$. For positive integers $n$ and $m$ with $n>m$,

$$
\begin{aligned}
\left\|\frac{1}{4^{m d}} f\left(2^{m} x_{1}, \cdots, 2^{m} x_{d}\right)-\frac{1}{4^{n d}} f\left(2^{n} x_{1}, \cdots, 2^{n} x_{d}\right)\right\| \leq \sum_{j=m}^{n-1} \sum_{i=1}^{d} \frac{1}{4^{i+j d}} \\
\quad \times \varphi\left(2^{j+1} x_{1}, 0, \cdots, 2^{j+1} x_{i-1}, 0,2^{j} x_{i}, 2^{j} x_{i}, 2^{j} x_{i+1}, 0, \cdots, 2^{j} x_{d}, 0\right),
\end{aligned}
$$

which tends to zero as $m \rightarrow \infty$ by (i). So $\left\{\frac{1}{4^{n d}} f\left(2^{n} x_{1}, \cdots, 2^{n} x_{d}\right)\right\}$ is a Cauchy sequence for all $\left(x_{1}, \cdots, x_{d}\right) \in \prod_{i=1}^{d} \mathcal{B}_{i}$. Since $\mathcal{D}$ is complete, $\left\{\frac{1}{4^{n d}} f\left(2^{n} x_{1}, \cdots, 2^{n} x_{d}\right)\right\}$ converges for all $\left(x_{1}, \cdots, x_{d}\right) \in \prod_{i=1}^{d} \mathcal{B}_{i}$. We can define a mapping $M: \prod_{i=1}^{d} \mathcal{B}_{i} \rightarrow$ $\mathcal{D}$ by

$$
M\left(x_{1}, \cdots, x_{d}\right)=\lim _{j \rightarrow \infty} \frac{1}{4^{j d}} f\left(2^{j} x_{1}, \cdots, 2^{j} x_{d}\right)
$$

for all $\left(x_{1}, \cdots, x_{d}\right) \in \prod_{i=1}^{d} \mathcal{B}_{i}$.

By (i) and (2), we get

$$
\begin{aligned}
& \left\|D M\left(x_{1}, 0, \cdots, x_{i-1}, 0, x_{i}, y_{i}, x_{i+1}, 0, \cdots, x_{d}, 0\right)\right\| \\
& \quad=\lim _{j \rightarrow \infty} \frac{1}{4^{j d}}\left\|D f\left(2^{j} x_{1}, 0, \cdots, 2^{j} x_{i-1}, 0,2^{j} x_{i}, 2^{j} y_{i}, 2^{j} x_{i+1}, 0, \cdots, 2^{j} x_{d}, 0\right)\right\| \\
& \leq \lim _{j \rightarrow \infty} \frac{1}{4^{j d}} \varphi\left(2^{j} x_{1}, 0, \cdots, 2^{j} x_{i-1}, 0,2^{j} x_{i}, 2^{j} y_{i}, 2^{j} x_{i+1}, 0, \cdots, 2^{j} x_{d}, 0\right) \\
& \quad=0
\end{aligned}
$$

for all $\left(x_{1}, \cdots, x_{d}\right) \in \prod_{i=1}^{d} \mathcal{B}_{i}$ and all $y_{i} \in \mathcal{B}_{i}$. Hence

$$
D M\left(x_{1}, 0, \cdots, x_{i-1}, 0, x_{i}, y_{i}, x_{i+1}, 0, \cdots, x_{d}, 0\right)=0
$$


for all $\left(x_{1}, \cdots, x_{d}\right) \in \prod_{i=1}^{d} \mathcal{B}_{i}$ and all $y_{i} \in \mathcal{B}_{i}$, which implies that $M$ is quadratic for each $i=1, \cdots, d$. Moreover, by passing to the limit in (1) as $n \rightarrow \infty$, we get the inequality (iii).

Now let $L: \prod_{i=1}^{d} \mathcal{B}_{i} \rightarrow \mathcal{D}$ be another multi-quadratic mapping satisfying

$$
\left\|f\left(x_{1}, \cdots, x_{d}\right)-L\left(x_{1}, \cdots, x_{d}\right)\right\| \leq \widetilde{\varphi}\left(x_{1}, x_{1}, \cdots, x_{d}, x_{d}\right)
$$

for all $\left(x_{1}, \cdots, x_{d}\right) \in \prod_{i=1}^{d} \mathcal{B}_{i}$. Then

$$
\begin{aligned}
\left\|M\left(x_{1}, \cdots, x_{d}\right)-L\left(x_{1}, \cdots, x_{d}\right)\right\|= & \frac{1}{4^{j d}}\left\|M\left(2^{j} x_{1}, \cdots, 2^{j} x_{d}\right)-L\left(2^{j} x_{1}, \cdots, 2^{j} x_{d}\right)\right\| \\
\leq & \frac{1}{4^{j d}}\left\|M\left(2^{j} x_{1}, \cdots, 2^{j} x_{d}\right)-f\left(2^{j} x_{1}, \cdots, 2^{j} x_{d}\right)\right\| \\
& +\frac{1}{4^{j d}}\left\|f\left(2^{j} x_{1}, \cdots, 2^{j} x_{d}\right)-L\left(2^{j} x_{1}, \cdots, 2^{j} x_{d}\right)\right\| \\
\leq & \frac{2}{4^{j d}} \widetilde{\varphi}\left(2^{j} x_{1}, 2^{j} x_{1}, \cdots, 2^{j} x_{d}, 2^{j} x_{d}\right),
\end{aligned}
$$

which tends to zero as $j \rightarrow \infty$ by (i). Thus $M\left(x_{1}, \cdots, x_{d}\right)=L\left(x_{1}, \cdots, x_{d}\right)$ for all $\left(x_{1}, \cdots, x_{d}\right) \in \prod_{i=1}^{d} \mathcal{B}_{i}$. This proves the uniqueness of $M$. This completes the proof.

Theorem 3. Let $f: \prod_{i=1}^{d} \mathcal{B}_{i} \rightarrow \mathcal{D}$ be a mapping for which there exists a function $\varphi: \prod_{i=1}^{d} \mathcal{B}_{i}^{2} \rightarrow[0, \infty)$ such that

$$
\begin{gathered}
\widetilde{\varphi}\left(x_{1}, y_{1}, \cdots, x_{d}, y_{d}\right):=\sum_{j=0}^{\infty} \sum_{i=1}^{d} 4^{i-1+j d} \varphi\left(\frac{1}{2^{j+1}} x_{1}, 0, \cdots, \frac{1}{2^{j+1}} x_{i-1}, 0\right. \\
\left.\frac{1}{2^{j+1}} x_{i}, \frac{1}{2^{j+1}} y_{i}, \frac{1}{2^{j}} x_{i+1}, 0, \cdots, \frac{1}{2^{j}} x_{d}, 0\right)<\infty, \\
\left\|D f\left(x_{1}, y_{1}, \cdots, x_{d}, y_{d}\right)\right\| \leq \varphi\left(x_{1}, y_{1}, \cdots, x_{d}, y_{d}\right)
\end{gathered}
$$

for all $\left(x_{1}, \cdots, x_{d}\right),\left(y_{1}, \cdots, y_{d}\right) \in \prod_{i=1}^{d} \mathcal{B}_{i}$. Assume that $f\left(x_{1}, \cdots, x_{d}\right)=0$ if $x_{i}=$ 0 for any $i=1, \cdots, d$. Then there exists a unique multi-quadratic mapping $M$ : $\prod_{i=1}^{d} \mathcal{B}_{i} \rightarrow \mathcal{D}$ such that

$$
\left\|f\left(x_{1}, \cdots, x_{d}\right)-T\left(x_{1}, \cdots, x_{d}\right)\right\| \leq \widetilde{\varphi}\left(x_{1}, x_{1}, \cdots, x_{d}, x_{d}\right)
$$

for all $\left(x_{1}, \cdots, x_{d}\right) \in \prod_{i=1}^{d} \mathcal{B}_{i}$.

Proof. The proof is similar to the proof of Theorem 2.

\section{REFERENCES}

1. C. Park, Multilinear mappings in Banach modules over a $C^{*}$-algebra, preprint.

2. Th.M. Rassias, On the stability of the linear mapping in Banach spaces, Proc. Amer. Math. Soc. 72 (1978), 297-300. MR 80d:47094

Department of Mathematics, Chungnam National University, DaeJeon 305-764, South Korea

E-mail address: cgpark@math.cnu.ac.kr 